Voix et Images

\title{
Le Temps des hommes d'André Langevin. Une relecture
}

\section{François Gallays}

Volume 1, numéro 3, avril 1976

Gérard Bessette

URI : https://id.erudit.org/iderudit/200040ar

DOI : https://doi.org/10.7202/200040ar

Aller au sommaire du numéro

Éditeur(s)

Les Presses de l'Université du Québec

ISSN

0318-9201 (imprimé)

1705-933X (numérique)

Découvrir la revue

Citer cet article

Gallays, F. (1976). Le Temps des hommes d'André Langevin. Une relecture. Voix et Images, 1(3), 406-416. https://doi.org/10.7202/200040ar d'utilisation que vous pouvez consulter en ligne.

https://apropos.erudit.org/fr/usagers/politique-dutilisation/ 


\section{Le Temps des hommes d'André Langevin Une relecture}

Des trois premiers romans d'André Langevin, le troisième et, croyaiton, le dernier - jusqu'à la publication de l'Élan d'Amérique en 1972 - ne semble pas avoir suscité autant d'intérêt chez les critiques québécois que l'avaient fait les deux premiers. A quoi cela peut-il tenir? Le roman est-il moins bien réussi que les précédents ? Peut-être. Cependant, pour ma part, je crois que la réponse se trouve ailleurs. Les lourdes traces, trop évidentes, que laissèrent les lectures existentialistes de l'auteur dans Évadé de la nuit et dans Poussière sur la ville permirent, encouragèrent même, leur déchiffrement à l'aide d'une grille philosophique analogue. Tel n'est plus le cas pour /e Temps des hommes.

Bien sûr, l'argument philosophique y est toujours, mais il n'apparaît pour ainsi dire presque jamais au premier plan et lorsqu'il perce à la surface du récit, c'est par l'entremise d'une modification dans la forme romanesque. C'est dire que du point de vue du lecteur cela s'effectue en quelque sorte naturellement. De plus, la distance que Langevin a su créer entre le postulat philosophique et le roman qu'il sous-tend laisse subsister certaines ambiguités qui rendent malaisée une interprétation univoque du roman ${ }^{1}$. Ces ambiguïtés sont à tel point profondes qu'elles permettent des interprétations non seulement divergentes, mais proprement contradictoires. L'interprétation que donne Simone Voisine du récit demeure sans doute la plus communément acceptée:

Pierre Dupas ne parvient apparemment pas à rejoindre Laurier qui tue Gros-Louis. Le "curé» poursuit sa recherche de Dieu; sa prière auprès du cadavre de Gros-Louis marque le point culminant du combat intérieur de l'homme qui revient à son Dieu. Sa démarche se clôt par une fidélité totale à Laurier qui a besoin de lui lors de sa fuite de la justice. Dupas retrouve donc Dieu dans une charité pour l'homme qu'il avait choisi d'aider en renonçant à Dieu? 2 .

Fin édifiante s'il en est puisque Dupas, le curé, réussit sur les deux plans: d'une part, il rejoint les hommes par l'entremise de Laurier et, d'autre part, il retrouve Dieu. La longue quête de Dupas se terminerait par une réussite totale - du moins par rapport à son projet original - car il aurait enfin atteint le but recherché pendant si longtemps: la jonction effective entre 
I'homme et Dieu. Cette interprétation, tout à fait satisfaisante évidemment sur le plan d'une certaine morale, doit laisser trop de choses dans l'ombre pour être pleinement acceptable. Gérard Bessette, dans son résumé du roman, fait entendre un autre son de cloche:

Ramené à Scottsville pour subir une amputation Dupas n'aura pas réussi à se rapprocher des hommes et il refusera toujours l'amour de Marthe ${ }^{3}$.

Pour Bessette, qui escamote complètement l'éventuel retour à Dieu de Dupas, le Temps des hommes se termine par l'échec du curé auprès des hommes. On le voit, l'interprétation de Bessette se trouve tout à l'opposé de celle de Simone Voisine. Ces divergences dans l'interprétation générale du roman peuvent être attribuées, évidemment, à une lecture quelque peu partielle... et partiale qui privilégierait certains événements au détriment d'autres jugés moins importants. Cependant, il est possible que cela tienne aussi au fait que, contrairement à ce qui se passe dans ses deux premiers romans, le romancier laisse planer ici sur l'accomplissement de gestes d'importance une ambiguïté qu'il n'a pas cherché à dissiper par le commentaire soit du narrateur ou du personnage impliqué. En d'autres mots, il se peut que l'auteur à certains endroits, n'ait pas jugé bon de fournir les indices aptes à motiver, ici une réplique, là un geste.

De toutes façons, et quelles que soient les raisons que l'on retienne pour justifier ces interprétations, celles-ci, parce qu'elles sont mutuellement exclusives, m'autorisent à produire, parallèlement, ma propre lecture du roman.

Frais émoulu du séminaire, Pierre Dupas, devant la souffrance et la mort d'un enfant, crie à l'injustice. Protestation spontanée, ce cri devient, conséquemment, une négation du mystère de la rédemption, pierre angulaire de la foi chrétienne. Cela, de toute évidence, revient à retirer à la mort du Christ sa valeur rédemptrice mais aussi sa valeur exemplaire sur laquelle se fonde l'explication et la justification de toutes morts innocentes. Entre le Dieu éternel et l'enfant qui souffre et qui meurt vraiment, Dupas, dans un moment de lucidité fulgurant, choisit le temps des hommes. Ayant rejeté le moyen unique qui permet aux hommes l'accession au monde divin, Dupas, devant la gravité de sa faute, quitte son ministère sacerdotal pour se réfugier en forêt, vivant tantôt en solitaire, tantôt auprès des bûcherons. Cherchant depuis dix ans à réapprendre "l'alphabet humain ${ }^{4}$ "auprès de ces hommes frustes mais, croit-il, authentiques et dont il n'a pas encore réussi à percer l'armure, Pierre Dupas attend le moment qui lui permettra de se racheter. Son but est donc double: d'une part, se rapprocher des hommes et, d'autre part, le moment propice, se racheter aux yeux de Dieu, c'est-à-dire poser le geste qui lui permettra de rétablir le lien entre les hommes et Dieu et, du même coup, sceller sa propre réconciliation avec son 
Créateur, effaçant ainsi le terrible blasphème proféré devant l'agonie scandaleuse de l'enfant. On le verra, cette réconciliation, contrairement à ce qu'affirme Simone Voisine ${ }^{5}$, ne se fera jamais. Le roman entier constitue, à mon avis, une sorte de preuve, par l'exemple, de l'impossible réconciliation de Dieu avec l'homme dans sa totalité.

Tout le long du roman, Langevin, par divers moyens, soulignera l'étanchéité de la cloison qui sépare Dieu des hommes. Ayant choisi de se mettre au service de Dieu, Pierre Dupas, dès le début de ses études au séminaire, dut subir un "enseignement qui l'obligeait à se détacher des hommes, qui éclairait de couleurs violentes et sombres les mots souffrance, péché et âme. Le temporel qui seul l'émouvait ne comptait plus pour rien, n'était plus que poussière dans une économie éternelle». (TH, p. 50) Responsables de l'enseignement religieux, les maîtres du séminaire, préoccupés par l'âme seulement, traitaient celle-ci comme une sorte de "prélèvement de laboratoire, un bouillon de culture isolé de son milieu». (TH, p. 51)

L'éducation du séminariste reposait sur une double série de valeurs antinomiques, l'une étant négative et dévalorisée, l'autre positive et recevant une survalorisation. D'un côté, se rangent l'âme, la pureté et l'éternité, de l'autre, le corps, le mal et la temporalité. Que tel fut en réalité l'enseignement dispensé dans les séminaires à l'époque où Langevin rédigea son roman, cela, en toute vérité, importe peu. Ce qu'il faut soùligner ici, c'est que la description de l'enseignement au séminaire par l'auteur, traduit une façon de voir et de juger les institutions et les valeurs qu'elles transmettent plutôt que de rendre compte fidèlement de la réalité. Et d'après Langevin, un abîme sépare Dieu des hommes. Selon le point de vue qu'il présente, on ne peut choisir les deux en même temps. Cela est de nouveau confirmé par les paroles de Monseigneur Major à qui Langevin fait dire que le prêtre, le "vrai prêtre se reconnaissant dans la chambre d'un agonisant, c'était là que pour la première fois il choisissait entre Dieu et les hommes». (TH, p. 109)

Selon l'Église et son enseignement officiel, le prêtre, évidemment, n'a pas à choisir Dieu contre les hommes puisque, de par sa fonction même, il lui revient, à lui ministre du culte, d'effectuer le lien entre les hommes et leur Dieu; c'est lui le trait d'union qui assurera, sa vie durant, la liaison entre le temporel et l'éternel.

Monseigneur Major tient donc des propos assez étranges pour un homme d'Église. Faut-il rappeler ici que Monseigneur Major étant une création du romancier, ses paroles, on peut le croire, expriment la conviction profonde de Langevin: le prêtre ne peut pas ne pas choisir entre les hommes et Dieu ${ }^{6}$. Pourtant, à un moment précis du roman, Langevin semble se contredire.

Devant le cadavre de Gros-Louis, celui-ci descendu il y a quelques instants par Laurier, Pierre Dupas, qui ne put empêcher le meurtre, retrouve spontanément les gestes et les paroles de l'homme oint de Dieu. C'est la première occasion qui se présente à Dupas depuis dix ans où il trouve à 
exercer sa fonction de prêtre. Afin de bien souligner la situation d'exil spirituel qu'a vécue pendant tout ce temps Dupas, l'auteur décrit sa réintégration sacerdotale dans les termes d'une métamorphose élémentale:

Une eau chaude l'inondait qui abolissait les gens, une ancienne démission, un recul faisait fondre la glace accumulée durant tant de jours. II priait. II pouvait prier. Les mots n'étaient plus des mots seulement, mais un filet mince qui le reliait à Dieu, des ondes qui le faisaient vibrer, une communion. Humblement, sans plus sentir dans son corps une solidarité avec la vie, toute chaire apaisée et consentante, il remettait à Dieu. II exerçait son ministère. (TH, p. 146)

Derrière les expressions, thermiques et aquatiques, qui servent à exprimer'la reprise momentanée de l'activité sacerdotale de Dupas, se profile, assez lourdement d'ailieurs, le retour symbolique de l'enfant au sein de sa mère, retour qui d'une façon générale se révèle comme la négation de la vie même?. Il devient vite évident, cependant, que l'acquisition de cette sécurité spirituelle est accompagnée par une perte vitale. L'avantdernière phrase du texte précité me paraît de ce point de vue très éloquente: «Humblement, sans plus sentir dans son corps une solidarité avec la vie ${ }^{8}$, toute chair apaisée et consentante, il remettait à Dieu. Il exerçait son ministère." (TH, p. 146) Ce n'est qu'en renonçant à la vie du corps, à la complicité viscérale du corps et de la vie, que Dupas peut remettre à Dieu.

A noter aussi, car cela me paraît avoir son importance, que Dupas redécouvre la voie qui conduit à Dieu seulement lorsqu'il se trouve à genou devant un cadavre. Si l'on rapproche ce geste de celui qui dix ans auparavant avait été provoqué par l'agonie de l'enfant, on doit conclure que, dans l'esprit de Langevin, il existe une incompatibilité irréductible entre la vie, la vie de l'homme, et toutes activités spirituelles commandées par la croyance en l'Être suprême. D'ailleurs le narrateur, en faisant mine de traduire la pensée du curé Dupas (mais on sent ici la présence de l'auteur), n'affirme-t-il pas que si celui-ci est retourné à Dieu, c'est bien parce qu'il avait échoué lamentablement auprès des hommes: "Il revenait à Dieu parce qu'il n'avait pas réussi avec les hommes. II revenait à Dieu parce qu'il restait seul avec Lui, " (TH, p. 147)

Revenu à Dieu, du moins momentanément, le curé n'a pas réussi, cependant, à rétablir le lien, rompu par son refus de jadis, entre Lui (Dieu) et les hommes, parce qu'il n'a pas su rejoindre ceux-ci. Il lui faut donc la confession de Laurier. De prime abord, cela peut paraître quelque peu étrange. Mais à la réflexion, il n'est que dans la logique des choses qu'un refus du rôle rédempteur du Fils de Dieu se rachète par l'acceptation de ce même rôle tel qu'inscrit dans le sacrement de pénitence. Laurier, que la jalousie et la haine ont rendu très lucide, perce les motifs de Dupas:

Je suis seul, curé, même avec toi et je veux le rester. Mon crime, c'est à moi. Tu tournes autour de moi comme si tu voulais me l'arracher. Je le tiens bien. Je mourrai avant de te le passer. (TH, p. 177)

La brutalité de ces paroles a pour effet de démasquer l'entreprise de Dupas à ses propres yeux. De noblè et de pure qu'elle fut, elle lui apparaît soudain comme un vulgaire acte d'échange, acte auquel Laurier 
refuse de se prêter. En rejetant la solution "spirituelle" que lui offre Dupas, Laurier exprime la volonté ferme d'assumer, au niveau des hommes, la mort de Gros-Louis. Sa charge d'humanité, quelle qu'en soit la qualité, Laurier entend la porter jusqu'au bout. Devant une telle attitude, paradoxalement empreinte de courage et de noblesse, étant donné qu'il s'agit de Laurier, Dupas reçoit la certitude que le rapport de forces entre eux vient de subir une modification majeure:

II n'y avait plus d'autre voie que celle de l'humiliation, de l'inutilité. Laurier avait une force d'âme dont lui-même était dépourvu. II ne pourrait plus que mendier. (TH, p. 184)

Désormais, Dupas, en témoin muet et impuissant, ne fera qu'accompagner Laurier qui, mû par une quelconque force instinctuelle, quasi animale, se lance dans une course folle à travers la forêt. Mais il y a plus. $A$ partir de ce moment, non seulement Dupas est-il réduit au silence et à l'impuissance (au niveau spirituel, bien sûr), non seulement a-t-il perdu toute initiative mais, subjugué par une force morale supérieure à la sienne et réduit, en conséquence, à la mendicité, il se voit, en quelque sorte, forcé à remettre entièrement son sort entre les mains de Laurier. Le lecteur assiste à ce moment précis du récit à une véritable prise en main du destin de Dupas par Laurier et ce, malgré l'affaiblissement physique de ce dernier.

Le long détour dans la forêt, que tentent d'accomplir les deux hommes en plein blizzard, parce que Laurier, ivre de jalousie, veut revoir, une dernière fois au moins, sa femme Yolande, ce détour, dis-je, prend peu à peu une signification toute symbolique. En ce qui concerne Laurier, il devient vite évident que celui-ci est en train d'effectuer son voyage ultime. Les indices semés par l'auteur en cours de route sont suffisamment nombreux pour que le lecteur ait très vite la certitude que Laurier s'achemine, à moins d'un miracie, directement vers sa mort. Seuls le moment et la manière demeurent voilés jusqu'à la toute fin. Ce voyage se transforme donc en un destin tragique en train de s'accomplir.

Pour ce qui est du prêtre, les choses se passent tout autrement. D'une certaine façon, le parcours symbolique de Dupas s'accomplit en sens inverse. En sortant de ce temps pour ainsi dire suspendu dans lequel il vécut dix ans, période de non-vie ${ }^{9}$, Dupas, grâce à Laurier, accède à la vraie vie, c'est-à-dire au temps des hommes. Ce passage qui ressemble en tous points à une véritable conversion, s'annonce d'abord par l'évacuation hors de l'esprit de Dupas de toutes préoccupations spirituelles. Face à l'agression de la nature qui le menace de mort, Dupas se transforme en pure résistance, ne se définit plus que par cette lutte primordiale qu'il mène pour sa survivance et celle de Laurier:

Il était épuisé lui aussi, vidé de toute révolte, habité par le vide [...]. Dupas n'avait même plus pitié. Il avait une tâche à accomplir, mécanique presque, qui était de soutenir Laurier, de le relever chaque fois qu'il tomberait. II l'acceptait, se concentrait entièrement sur cette tâche, refusait de s'en laisser distraire par les mots. (TH, p. 184)

Devant l'existence menacée,., la sienne et celle de Laurier, Dupas, d'une façon instinctive, met toutes ses énergies au service de la préserva- 
tion de la vie. C'est au niveau le plus élémentaire que s'accomplit son apprentissage de l' “alphabet humain ". Autre chose, à mesure que la menace de mort se précise, ce vide intérieur, créé par la lutte que mène Dupas au niveau des corps, sera bientôt meublé par un sentiment bien humain lui aussi : celui de l'angoisse devant l'imminence de sa propre mort :

Il ferma les yeux et la panique lui étreignit le cœur. II allait mourir ainsi, allongé à côté de Laurier, mourir de froid, de faim, enseveli sous la neige. (TH, p. 187)

Exténué, incapable de réagir malgré sa panique, Dupas commence à s'abandonner au sommeil aux côtés de Laurier lorsque, soudain, dans tout son corps, c'est-à-dire toujours au niveau de l'instinct, il reçoit un «avertissement violent $"$ :

Laurier le mettait en joue et essayait pitoyablement de lui dire quelque chose d'essentiel. Il avait l'âme aux bords des yeux, mais rien ne passait de son message. Dupas n'obéit qu'à sa terreur. II saisit l'automatique et le détourna de lui. (TH, p. 188)

Par un geste d'auto-conservation, surgissant du pur instinct, à situer donc, au niveau de l'humain le plus élémentaire, Dupas échappe à la mort. Mais du même coup il tue Laurier.

Malgré son caractère accidentel, la mort de Laurier révèle très clairement que Dupas a préféré instinctivement sa propre vie à celle de Laurier. Et cela me paraît capital. Car c'est à ce moment précis que Dupas, grâce à son geste, rejoint d'emblée l'humain. Plus. II se met à ressembler à ces hommes parmi lesquels il a vécu pendant dix ans et qui appartiennent à cette part d'humanité réduite à sa plus simple expression: “Des exemplaires d'hommes sans camouflage, réduits à l'essentiel.» (TH, p. 48) Ces hommes n'obéissent à tous les niveaux de leur vie - mais cela se révèle surtout au niveau affectif - qu'à des pulsions instinctuelles primaires, vivant pour ainsi dire par et pour leur corps: "Leurs corps lourds requéraient tous leurs soins." (TH, p. 49) Sous cet aspect, on doit donc les situer aux antipodes des hommes formés dans les séminaires et à qui on demandait de renier pour toujours le corps et certaines de ses exigences vitales: "Nous ne sommes pas de ce monde". Cette phrase à laquelle il avait si ardemment cru, dure, d'un orgueil sublime." (TH, p. 34)

Que Dupas ait enfin intégré le temps humain, qu'il ait, par conséquent, subi une transformation sur le plan humain, la conclusion en fournit la preuve lorsque curieusement, du moins en apparence, ce n'est pas Marthe mais Yolande qui embrasse Dupas sur la bouche. Pour bien saisir la signification de ce geste qui clôt le roman, il faut rappeler le jugement que porte Yolande sur Dupas au tout début du récit:

Yolande l'avait toujours regardé avec indifférence. Elle savait sans comprendre pourquoi, qu'il était de ces hommes qu'elle n'embrasserait jamais. Il lui suffisait d'une seconde pour classer les hommes dans l'une ou l'autre catégorie. (TH, p. 13)

Profondément enracinée dans le charnel, femelle jusque dans ses moindres gestes, Yolande, intuitivement et très sûrement, sait repérer les hommes qui 
refusent la vie du corps comme aussi ceux qui l'acceptent. A la fin du roman Yolande reconnaît en Dúpas un homme qui est passé de son côté.

On pourrait, évidemment, expliquer le baiser de Yolande par le phénomène de transfert, bien connu en psychanalyse, mais cette explication, loin d'infirmer la portée symbolique de l'acte, la confirmerait. Dupas, aux yeux de Yolande, peut maintenant sans démériter souffrir la comparaison avec Gros-Louis. Symboliquement, donc, le baiser de Yolande ne peut que signifier l'intégration de Dupas au monde des hommes et ce, même s'il est physiquement diminué.

Ainsi, dans la mythologie personnelle de Langevin, du moins telle qu'elle se développe dans le Temps des hommes; on devient homme, on accède au temps des hommes par le biais d'une confrontation (rite initiatique?), avec la nature et ses éléments et avec ses semblables. Ce combat, Langevin le situe au niveau élémentaire de la survivance, car c'est là, semble-t-il, que se prend la véritable mesure des hommes, étant donné que c'est à ce niveau critique que perce et s'impose le fonctionnement instinctuel de tout un chacun.

Contrairement donc à ce qu'affirme Bessette (voir supra), la trajectoire de Dupas n'aboutit pas à un double échec. Est-ce à dire, cependant, comme l'affirme Simone Voisine (voir supra) que Dupas a enfin réussi la jonction entre Dieu et l'humain? Non pas. Par un retournement ironique du sort, Dupas se trouve à faire périr celui sur la vie de qui il avait misé toutes ses chances pour retourner au monde divin:

... il but longtemps pour maintenir en lui la flamme, pour effacer dix années de froid, pour jeter un pont par-dessus les dix années, entre l'enfant et Laurier, entre sa ferveur d'autrefois et sa tiédeur d'aujourd'hui. (TH, p. 124)

Or, c'est de ses propres mains que Laurier trouve la mort... À n'en pas douter, la mort de Laurier, étant donné son contenu ironique, traduit très fidèlement, à mon avis, la pensée profonde de l'auteur, à savoir que l'univers des hommes et l'univers de Dieu sont antinomiques. Réciproquement exclusifs, ils ne sauraient être habités par quiconque simultanément. Ainsi Dupas passe du côté des hommes grâce au geste qui l'éloigne à jamais, dirait-on, de la bienfaisance divine. Mais ce même geste, de toute évidence primordiale pour Dupas, confère aussi à Laurier une dimension nouvelle.

Laurier, homme parmi les hommes s'il en fut, est mort afin que Dupas, ministre de Dieu, quitte enfin ce monde raréfié du sacerdoce pour entrer de plain-pied dans l'univers de l'homme. Par le "rachat " de la vie du prêtre dont elle est reponsable, la mort de Laurier renvoie parodiquement à la mort du Christ. Cette nouvelle figure, de nature christique, que la mort sacrificielle donne à Laurier, se trouve à prendre du relief si, rétrospectivement et analogiquement, l'on effectue un rapprochement entre le chemin qui mena le Christ jusqu'à son calvaire et cette interminable course en forêt qui conduisit Laurier à sa mort. Toujours par le biais rétrospectif, on s'aperçoit, de plus, que Laurier n'a pas seulement agi comme "sauveur" auprès de Dupas, mais aussi, par un renversement des rôles, comme un 
"prêtre", c'est-à-dire celui qui peut recevoir la confession. Ainsi, l'entretien, durant lequel Dupas dévoile ses véritables antécédents, reçoit de Laurier le nom de confession:

On a bien du temps devant nous. Raconte un peu, curé. Les prêtres aussi vont à confesse. Tiens, bois. Ça aide. (TH, p. 100)

De confesseur, Dupas devient le confessé, le pénitent, tandis que Laurier s'arroge le rôle de ministre. Amorce d'une nouvelle relation entre les deux hommes, la confession de Dupas marque le début de l'ascendant total que finira par exercer Laurier sur le prêtre. II faut de plus ajouter que la confession de Dupas constitue aussi le seul retour en arrière du roman. C'est dire qu'au renversement des rôles au niveau des personnages correspond une rupture dans la composition du roman, soulignant ainsi le coup de barre donné à la direction de l'action précisément à cet endroit. Mais ce retour en arrière joue aussi un rôle de première importance dans la structuration du roman, ainsi que les décès qui lui donnent sa ponctuation.

La mort de Laurier clôt la série paradigmatique des trois décès que contient le roman ${ }^{10}$. Cependant, à cette série vient s'ajouter une quatrième mort qui, même si elle ne figure pas comme telle dans le roman, y occupe néanmoins, par une sorte de présence immanente, une place importante. II s'agıt, mais on l'aura déjà deviné, de la mort du Christ. Ce n'est, en effet, que par leur mise en relation avec celle-ci que les trois morts du roman revêtent leur pleine et entière signification. Par exemple, si la mort de l'enfant provoque chez Dupas une sorte de syncope qui, en détruisant son univers jusque-là sans failles, le voue à l'errance parmi les hommes, c'est parce que le jeune curé fut incapable d'accepter la valeur rédemptrice de la mort du Christ, celle qui, censément, justifie toutes les morts innocentes. Le refus de la mort de l'enfant, Dupas l'envisagea comme une sorte d'échec personnel auprès de Dieu, échec qu'il promit de réparer.

Impuissant auprès de Dieu, Du:pas le sera aussi auprès des hommes, car il ne pourra pas empêcher la mort de Gros-Louis. II est réduit à réciter des prières au-dessus du cadavre alors qu'il voulait que solt épargnée la vie du bûcheron. Ce n'est que par la mort de Laurier, dont il se trouve indirectement responsable, que s'ouvre la voie vers les hommes.

On le constate, parce que chacune des morts occupe un point d'artıculation dans le récit de la vie de Dupas, elles doivent être envisagées, sur le plan de la narration, comme autant de noyaux autour desquels se construit le récit. Les considérant de ce point de vue, on ne saurait évidemment néglıger le rôle de la mort du Christ qui, rappelons-le, est à situer au sommet de la série.

En schématisant quelque peu, celle-ci s'articule de la façon suivante:

Mort I (du Christ): Ouverture sur le ciel

Mort II (de I enfant): Échec auprès de Dieu

Mort III (de Gros-Louis) : Échec auprès des hommes

Mort IV (de Laurier): Ouverture sur le monde humain. 
Ainsi, donc, un "événement " ne faisant pás partie du texte, ne se trouvant nulle part exprimé en clair, est appelé, néanmoins, à jouer un rôle fondamental dans sa structuration puisque c'est grâce à cet "événement " extérieur que sont signifiants les éléments structurant le récit.

Si maintenant on place les quatre éléments de la série ci-dessus sur l'axe temporel, on s'aperçoit qu'une coupure très nette s'impose entre Mort I et II, d'une part, et Mort III et IV, d'autre part, dans la mesure où les deux premiers éléments de la série appartiennent à un temps antérieur au récit, tandis que les deux derniers s'inscrivent dans le temps discursif du roman. Qui plus est, cette antériorité est inscrite dans la forme même du roman du fait que celle-ci se retrouve dans le seul retour en arrière du roman, segment qui, étant donné son autonomie, constitue une sorte de récit dans le récit. Véritable coupure dans le tissu du récit, le retour en arrière, parce qu'il est la relation d'une situation antérieure, permet au récit de développer une seconde situation qui se trouve à renverser complètement la première. L'on voit mieux maintenant l'importance fonctionnelle que revêt la mort du Christ: sa présence, subsumée dans la situation première, autorise l'attribution, dans la situation nouvelle, d'une fonction analogue, c'està-dire salvatrice, à la mort de Laurier.

Sur le plan humain, la valeur rédemptrice de la mort de Laurier ne fait aucun doute. Mais pourquoi Laurier? Pourquoi, serait-on en droit de demander, l'auteur a-t-il choisi précisément ce meurtrier pour "racheter" Dupas? pour le ramener, en quelque sorte, dans le «droit sentier» des hommes? A la réflexion, les raisons qui, de prime abord, font douter de la justesse du choix de Langevin sont celles-là mêmes qui le fondent et le justifient. Il a choisi Laurier justement parce que c'est un meurtrier, parce que c'est un homme capable des pires bassesses. Par ce choix, le romancier signifie que non seulement Laurier est-il homme malgré le caractère hautement répréhensible de ses actes, mais qu'essentiellement ce sont ces actes mêmes qui témoignent de son humanité, non exempté, en fin de compte, d'une certaine grandeur. Car, mû par la passion de jalousie, dont les exigences sont telles qu'elle le mènera finalement à sa perte, Laurier atteint, mais par le bas, à une sorte de grandeur tragique. Ce travestissement tragıque, ou plutôt, ce qui serait sans doute plus juste, cette parodie de la tragédie - caractérisation, selon moi, la plus adéquate du comportement du héros langevinien - est source génératrice d'un pathétique, révélateur, chez le romancier, d'une bienveillance qui me paraît s'étendre dans le Temps des hommes à tous les personnages.

Pour ce qui est de Dupas, celui-ci à l'instar de certains héros de contes populaires, après avoir "erré", c'est le cas de le dire, pendant une dizaine d'années en quête d'un idéal inaccessible (rappelons l'antinomie du couple humain/divin chez Langevin), et subi l'épreuve finale dont il sort victorieux, celui-ci est admis dans la société des hommes, mettant fin, de cette manière, à son aliénation.

Ce faisant, Dupas, en passant d'un monde à un autre, se sépare de la majorité des héros qui, ayant réussi à éviter la mort, reprennent leur place 
dans la société qu'ils avaient quittée avec l'espoir de la modifier selon les nouvelles valeurs qu'ils auront rapportées de leurs pérégrinations initiatiques. Si le romancier Langevin n'a pas imposé une fin analogue au Temps des hommes, c'est parce qu'il eût fallu qu'une compénétration des deux mondes en présence dans le roman soit possible. Or on sait que cela est contraire à la pensée langevinienne. Eu égard aux aspirations premières du héros, on pourrait envisager l'aboutissement de son action comme une demi-victoire seulement puisqu'il n'a atteint qu'une partie du bien désiré. Cependant, le geste instinctif de.Dupas, au moment du dénouement, fera éclater ce bien désiré du début et en révélera le caractère composite et contradictoire. À ce moment critique, le bien désiré se scinde en deux: d'un côté, le "bien» choisi instinctivement (sa propre vie), donc le bien désiré profondément, et de l'autre côté, le "bien" imposé par une éducation dualiste qui, en ignorant les pulsions profondes de l'homme, fait cuvre de mort. Autrement dit, ici, le monde et l'homme, c'est-à-dire la vie; là, le monde divin et la mort, c'est-à-dire le mal absolu.

On le voit, la lecture du Temps des hommes révèle un monde au départ profondément dichotomisé. À la fin, un pan s'est écroulé, n'est plus que fine poussière, tandis que l'autre tient bon malgré ses failles et ses fissures. A une époque (1956) où l'édifice religieux au Québec masque encore le cancer qui le ronge quand même depuis quelques années ; à une époque où l'Église du Québec est encore assez puissante pour imposer, à toute une population, sa définition de l'homme, Langevin s'insurge contre ce type d'appropriation en proposant une idée de l'homme radicalement autre parce que fondée sur une saisie totale du champ humain. Mais en redéfinissant l'homme, tel qu'on le retrouve dans le Temps des hommes, Langevin vise aussi, au-delà de l'Église, cet humanisme intellectualisant, universaliste, qui s'est toujours singularisé par une valorisation partielle, et à fortiori atomisante, de l'homme en ne reconnaissant dans sa définition de la personne humaine que les facultés dites nobles. Contre l'Église, et I'humanisme à sa remorque, qui offrent, à peu de choses près, la même vision de l'homme, se dresse un nouvel humanisme qui reconnaît et accepte l'être humain dans sa totalité; c'est-à-dire un être qui obéit aussi à ses pulsions, à ses instincts et à ses déterminismes.

Toutefois, l'humanité que présente et définit le romancier est très loin de l'espèce humaine, glorieuse et païenne, dont un Camus a chanté la beauté dans quelques-uns de ses textes. Tout le contraire. Chaque homme et chaque femme, que Langevin fait apparaître dans le Temps des hommes, souffre d'une faiblesse quelconque, d'une carence, d'un manque. Et il est à noter que c'est autour de ce manque que d'une façon générale se construit le personnage; c'est sur cette carence que repose la singularité du personnage. Songeons à Baptiste et à sa toux, à Maurice et à ses photographies obscènes, songeoons à Marthe et à son amour muet, à Laurier et à sa jalousie. Mais le meilleur exemple, c'est évidemment le curé Dupas qui, au moment où il entre dans le monde des hommes, doit se faire amputer les deux jambes. En plus, il est accueilli par une femme "naturellement impudique ", mais dont le corps est disgracieux de la taille en bas. 
II semble assez clair que le monde dans lequel Dupas fait son entrée est un monde qui vit entièrement sous le signe de la castration. L'absence pénienne, signalée, dans la majorité des cas, par l'impossible éclosion de la jouissance, se manifeste évidemment tant chez les femmes que chez les hommes. Même Gros-Louis, sorte de dieu fruste, qui ne paraît souffrir d'aucune faille, est malgré tout vulnérable, du moins métonymiquement si on peut s'exprimer ainsı, par le biais de sa sœur, gravement atteinte de leucémie et enfermée dans un couvent.

Le Temps des hommes se ferme donc sur un paradoxe. D'un côté, le roman reconnaît d'une façon explicite le caractère impérieux des courants pulsionnels qui traversent les hommes, tandis que de l'autre côté, est affırmée leur impossible éclosion. Autrement dit, le roman reconnaît dans son principe l'existence de l'énergie instinctuelle mais il a été incapable d'en reconnaître la finalité. La résolution du paradoxe n'étant pas contenue dans le roman, il faudrait la chercher ailleurs. Chez le romancier? Sans doute. Mais ceci est un autre problème.

François Gallays Université d'Ottawa

1. Pour cette raison, la même grille d'interprétation appliquée aux trois romans ne saurait donner dans chaque cas le même résultat. Je ne prendrai comme exemple que l'article de Jean-Louis Major consacré à André Langevin dans le Roman canadien-français (Montréal, Fides, 1964) où la grille adoptée, bien que suffisante pour une interprétation globale - du moins d'un certain point de vue - des deux premiers romans, n'a pu que faire ressortir quelques éléments secondaires dans le Temps des hommes tout en laissant de cóté l'essentiel.

2. Simone Voisine, "l'Expression de l'espace dans la trilogie romanesque d'André Langevin ", dans Étudés littéraires, vol. 6, n², août 1973, p. 211.

3. Gérard Bessette, “l'Elan d'Amérique dans l'œuvre d'André Langevin ", dans Livres et auteurs québécois 1972, Montréal, Jumonville, p. 18.

4. André Langevin, le Temps des hommes, Montréal, Le Cercle du livre de France, 1956, p. 70 . Ce roman sera dorénavant désigné par le sigle TH.

5. Voir supra.

6. On peut constater ici l'habileté du romancier. Langevin, en se servant de $\mathrm{M}^{9 \mathrm{r}}$ Major comme truchement, fait passer ce qui est une vision bien personnelle des relations entre Dieu et les hommes pour la position officielle de l'Église. Grâce à son personnage, il peut, avec vraisemblance, ériger en principe une dichotomie qui, dans la réalité, a peut-être existé, mais dont on a toujours nié l'existence au niveau théorique.

7. En poussant un peu plus loin cette réflexion, on pourrait affirmer que Dupas, devant la trop brutale réalité du meurtre, se retourne spontanément, en effectuant une sorte de régression, vers le monde de son enfance où la prière occupait une place importante puisqu'il fut éleyé par ce vieil abbé Pottier qui lui servit aussi bien de mère que de père. L'Église, donc, dans l'affectivité de Dupas orphelin, par voie de substitution assez évidente, aurait remplacé la mère absente. Ainsi la chaleur aquatique qui enveloppe tout l'être de Dupas au moment même où il se met à prier devant le cadavre de Gros-Louis serait la conséquence directe et immédiate du retour au monde sécurisant de sa tendre enfance.

8. C'est moi qui souligne.

9. C'est le sens méme de l'enseignement reçu au séminaire: “ Hors des murs vous n'appartenez ni à un côté, ni à l'autre. En suspens dans une zone de refus." (TH, p. 34)

10. C'est à dessein que j'exclus la mort de Baptiste. 